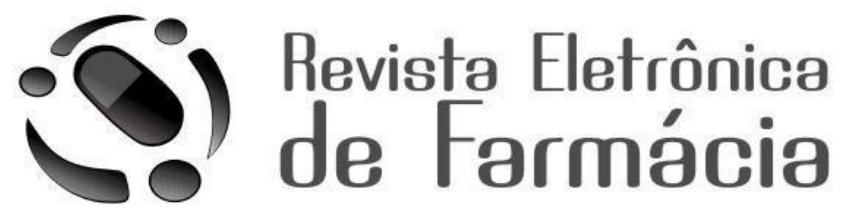

REF-ISSN1808-0804 Vol.XI (2), 55-69, 2014.

\title{
SEMIOLOGIA FARMACÊUTICA E OS DESAFIOS PARA SUA CONSOLIDAÇÃO
}

\author{
PHARMACEUTICAL SEMIOLOGY AND CHALLENGES TO ITS \\ CONSOLIDATION
}

\section{SEMIOLOGÍA FARMACÉUTICA Y RETOS PARA SU CONSOLIDACIÓN}

RESUMO: A semiologia farmacêutica pode ser entendida como a identificação de sinais e sintomas, principalmente aqueles relacionados aos transtornos/distúrbios menores relatados pelo paciente. Essa prática profissional não deve ser confundida com diagnóstico, que é uma atividade realizada pelo médico, mas como uma nova ferramenta na dispensação ativa de medicamentos de venda livre. Este estudo visa identificar o desenvolvimento dessa prática profissional e discutir os desafios necessários à sua implementação.

PALAVRAS-CHAVE: Semiologia farmacêutica; Doenças menores; Prescrição farmacêutica; Automedicação orientada; Medicamentos isentos de prescrição.

ABSTRACT: Pharmaceutical semiology can be comprehended as the identification of signs and symptoms, especially those related to minor disturbances/disorders reported by the patients. This practice should not be confused with diagnosis, which is an activity performed by the doctor, but as a new tool in the active dispensing of prescription drugs. This study aims to identify the development of this practice and discuss the challenges required for its implementation.

KEYWORDS: Semiology pharmaceutical; Minor illnesses; Prescription pharmaceuticals; Oriented self-medication; Over the counter medicine.

RESUMÉN: La semiología farmacéutica puede ser entendida como la identificación de los signos y síntomas, en especial los relacionados con los trastornos / alteraciones menores reportados por el paciente. Esta práctica profesional no debe ser confundido con el diagnóstico, que es una actividad realizada por el médico, sino como una nueva herramienta de dispensación en los medicamentos de venta libre. Este estudio tiene como objetivo identificar el desarrollo de esta práctica profesional y discutir los retos necesarios para su aplicación.

PALABRAS CLAVE: Semiología farmacêuticos; Enfermedades menores; Medicamentos con receta; Orientada a la automedicación; Medicamentos de venta libre.

Tahisa Marcela Pedroso, Patrícia de Carvalho Mastroianni, Jean Leandro dos Santos.

Recebido em 04/02/2014, Aceito em 29/04/2014. 
PEDROSO, T. M.; MASTROIANNI, P. C.; SANTOS, J. L.

REF-ISSN1808-0804 Vol.XI (2),55-69, 2014.

\section{INTRODUÇÃO}

De acordo com a Organização Mundial de Saúde - OMS, muitos são os fatores que desencadeiam o uso irracional de medicamentos, entre eles a carência de conhecimento do usuário e o acesso de muitos medicamentos que não necessitam de receita médica, permitindo o uso de medicamentos desnecessários e propiciando uma terapêutica duvidosa $^{(1)}$. Grande parte da população por não dispor de assistência médica com facilidade recorre à automedicação, buscando nas farmácias a resolução para os seus sintomas mais comuns. No entanto a automedicação quando feita sem a orientação adequada de um profissional da saúde pode provocar agravos que seriam minimizados com a implantação de dispensação ativa, onde o usuário recebe orientação farmacêutica adequada quanto ao uso seguro dos medicamentos, como indicação e forma correta de administração, os benefícios e riscos de sua utilização minimizando assim, o ônus sócio econômico nos diferentes níveis de atenção à saúde com atendimentos devido a agravamentos a saúde ${ }^{(2-3)}$.

No ano de 1992, o International Pharmaceutical Federation (FIP) elaborou um documento para padronizar as Boas Práticas de Farmácia, que abrange atividades para incentivar o auto-cuidado, incluindo o aconselhamento aos usuários e, quando apropriado, a indicação de tratamentos medicamentosos, para aliviar desconfortos e enfermidades leves. O farmacêutico deve estar atento ao histórico médico do usuário para dispensar medicamentos seguros, eficazes e de qualidade em colaboração com os outros profissionais $^{(4)}$.

A Associação Mundial de Médicos da Família (WONCA) entende que, um problema de saúde, é qualquer queixa, observação ou fato que o paciente ou o profissional percebam como um desvio da normalidade e que possa afetar a capacidade funcional do paciente. O farmacêutico é o profissional capaz de identificar, resolver e prevenir potenciais problemas relacionados a medicamentos (PRMs) com o uso racional de medicamentos e possíveis intervenções, em um plano terapêutico efetivo e seguro, através da educação do paciente frente aos medicamentos ${ }^{(5)}$. Estes recursos permitem otimizar a terapia medicamentosa, promovendo o bem estar e são capazes de reduzir PRMs melhorando a qualidade de vida dos usuários $^{(6)}$.

A semiologia farmacêutica é a aplicação dos conhecimentos previamente adquiridos, no qual 0 
PEDROSO, T. M.; MASTROIANNI, P. C.; SANTOS, J. L.

REF-ISSN1808-0804 Vol.XI (2),55-69, 2014.

farmacêutico como profissional da saúde utiliza a fim de resolver distúrbios menores relacionados à farmacoterapia. Os sinais e sintomas referidos pelo paciente devem ser observados a fim de esclarecer a natureza da doença e traçar os planos terapêuticos conjuntos com o paciente e com a equipe de saúde ${ }^{(7-}$ ${ }^{8)}$. Os sinais podem ser percebidos e quantificados, enquanto que os sintomas são percepções humanas e dependem do relato do paciente. A prática da semiologia farmacêutica é ainda pouco conhecida entre os profissionais de saúde por isso, é necessário agregar conhecimentos ao currículo do farmacêutico, inserindo desde a graduação a importância de prestar cuidados de saúde à sociedade, para que possam assim, implantá-la de forma segura ${ }^{(9)}$.

Em vista da importância da semiologia farmacêutica e de seu pouco conhecimento e aplicação, foi realizada uma revisão, com o objetivo de identificar estudos de semiologia farmacêutica em diferentes países, incluindo Brasil. Os principais sinais e sintomas, que incentivam os usuários de medicamentos a procurarem as farmácias para indicação de medicamentos para o tratamento dos distúrbios referidos e a automedicação responsável no tratamento de distúrbios menores também foi investigada e relatada neste estudo.

\section{METODOLOGIA}

Foi realizada uma busca nas bases de dados: Bireme, Embase, Lilacs, Medline/PubMed, Periódicos Capes, Scopus, ScienceDirect, Scielo e SciFinder, utilizando-se os seguintes descritores científicos em saúde: "Community pharmacy services", "Clinical interview", "Clinical pharmacist", "Drugstore", "Minor illnesses", "Nonprescription drugs", "Over-the-counter", "Pharmaceutical care", "Pharmaceutical semiology", "Pharmaceutical services", "Pharmacist counseling", "Primary care", "Self-medication", "Signs and Symptoms". Uma pré-análise dos artigos foi realizada a fim de identificar estudos de semiologia farmacêutica em diferentes países, incluindo Brasi.

. Foram considerados elegíveis para o estudo os manuscritos originais, redigidos em português, inglês ou espanhol, disponíveis com texto completo, que correlacionavam à ação farmacêutica com sinais e sintomas e/ou que avaliaram os serviços farmacêuticos realizados em farmácias e drogarias. Excluíram-se da pesquisa os artigos de revisão, pesquisas realizadas com pacientes simulados, trabalhos qualitativos, os editoriais, cartas ao leitor, resumos apresentados de anais de congressos 
PEDROSO, T. M.; MASTROIANNI, P. C.; SANTOS, J. L.

REF-ISSN1808-0804 Vol.XI (2),55-69, 2014.

e resultados de dissertação de mestrado ou tese de doutorado. 0 período para a busca de evidências decorreu de 1900 a 2014.

\section{RESULTADOS}

Um movimento de incentivo à Semiologia Farmacêutica nas instalação de cuidados primários para atender a população capaz de se "autocuidar" tem sido observado, essa mudança vem sendo apoiada por muitos países, para manter capacidade produtiva da população, melhorar a qualidade de vida e diminuir os custos com os cuidados de saúde, permitindo que os profissionais de saúde possam se concentrar em problemas mais graves ${ }^{(10-11)}$. Os termos "auto-cuidado" e "automedicação responsável" estão intimamente relacionados à semiologia farmacêutica. Em 1998 a Organização Mundial da Saúde - OMS no documento "The role of pharmacist in self care and self medication" ressalta a importância do farmacêutico na atenção primaria, auxiliando os pacientes na tomada de decisão por meio da orientação. Neste documento o autocuidado é definido como, o que as pessoas fazem por si mesmas para manter a saúde e prevenir as doenças. Nestas circunstâncias engloba a automedicação, que por sua vez é a seleção e uso de medicamentos para tratamento de enfermidades ou sintomas reconhecidos, pelo próprio individuo, e se difere da automedicação responsável, pois nesta, há o consumo de fármacos de venda livre aprovados, que quando usados como indicados são considerados seguros e eficazes ${ }^{(6)}$.

A automedicação quando realizada de maneira responsável, é necessária para complementar as funções dos sistemas de saúde, particularmente para os países em desenvolvimento $^{(12)}$. Em 1986, a Organização Mundial de Saúde já publicava diretrizes para a avaliação dos medicamentos que poderiam ser empregados na automedicação, os quais devem ser eficazes, confiáveis, seguros e de emprego fácil e cômodo $^{(13)}$. Com a expansão de medicamentos disponíveis sem receita médica, o farmacêutico assume um papel fundamental no aconselhamento ao paciente. Em muitos casos os pacientes são capazes de tomar decisões em relação a sua própria saúde, se este dispor de orientação especializada. Muitas pessoas querem liberdade para escolher os seus medicamentos e cabe aos farmacêuticos orientá-los para que elas façam a melhor opção baseadas nas informações e conselhos referidos pelo farmacêutico ${ }^{(11)}$.

A anamnese realizada pelo farmacêutico é essencial na prevenção de doenças primárias, no tratamento de doenças agudas de rotina e na 
REF-ISSN1808-0804 Vol.XI (2),55-69, 2014.

questão de doenças crônicas, para isso é necessário alcançar uma relação fidedigna com o paciente. Este é o principal veículo de informações sobre o seu estado de saúde e, portanto, representa a principal ferramenta utilizada para identificar os sinais e sintomas apresentados e correlacioná-los com possíveis enfermidades ${ }^{(14-15)}$.

Um estudo realizado na Estónia definiu a "doença menor" como um autocuidado que requer uma tomada de decisão, um processo que envolve a auto-observação, percepção dos sintomas e julgamento da gravidade para a escolha de um tratamento ${ }^{(11,16)}$. As doenças menores são autocondições limitantes que requerem pouca ou nenhuma intervenção médica, tais como: resfriados, tosses e indigestão, que podem ser tratadas com medicamentos isentos de prescrição (MIP). Apesar de serem considerados relativamente seguros, muitos MIPs também são conhecidos por conter agentes farmacológicos com potencial para causar reações adversas e interações medicamentosas, por isso a sua utilização exige o mesmo grau de cuidado que os medicamentos vendidos sob prescrição médica. No Reino Unido as estatísticas de morbidade sugerem que mais de $40 \%$ das consultas ao clínico geral são para doenças menores que poderiam ser tratados sem aconselhamento médico, mas com uso acompanhado por outros profissionais da saúde a exemplo dos farmacêuticos ${ }^{(16)}$.

A política do governo do Reino Unido destaca o papel das farmácias comunitárias no apoio seguro e eficaz de auto-cuidado, elas fornecem conselhos de saúde em geral e informações específicas sobre os medicamentos isentos de prescrição. $\mathrm{Na}$ Escócia a implantação de um serviço de atendimento a doenças menores nas farmácias comunitárias tem sido reforçada, permitindo que estes pacientes sejam tratados diretamente na farmácia(10). As campanhas farmacêutica como, "Escolha o remédio certo" e "pergunte ao seu farmacêutico", foram implementadas para encorajar os pacientes a solicitar orientações aos farmacêuticos. Este foi apenas um primeiro passo em uma ampla gama de serviços prestados por esses profissionais, incluindo assistência em doenças menores. Novas legislações foram então criadas para permitir que os farmacêuticos, (bem como outros profissionais de saúde) possam prescrever certos medicamentos para suprir a atenção primaria, baseandose sempre em protocolos rígidos, tais protocolos foram introduzidos pelo governo do Reino Unido em agosto de $2000^{(17)}$.

Em 2004, foi realizado um trabalho com refugiados asilados no sul de Londres, que por estarem 
PEDROSO, T. M.; MASTROIANNI, P. C.; SANTOS, J. L.

REF-ISSN1808-0804 Vol.XI (2),55-69, 2014.

afastados da atenção basica de saúde recebiam "tickets" que Ihes permitiam ter uma consulta com farmacêutico e troca-los por medicamentos isentos de prescrição (MIPs) gratuitamente, para tratar de suas doenças menores. Este estudo fez um levantamento das doenças mais frequentes nessa região e os fármacos mais dispensados para essas enfermidades. Indica que é possivel tratar as "doenças menores" com os fármacos de venda livre, evitando consultas médicas desnecessaria. É importante considerar que uma pequena parcela desses individuos foram encaminhados para unidade médica ${ }^{(18)}$.

Em 2007, uma revista americana já apontava uma tendência crescente para as consultas farmacêuticas e recomendações de MIPs nos cuidados de saúde, pois o farmacêutico é a pessoa mais próxima ao paciente para aconselhá-lo. Neste mesmo período uma pesquisa nos Estados Unidos confirmou que muitos farmacêuticos já estavam oferecendo este valioso serviço profissional. A pesquisa apontou que produtos isentos de prescrição mais recomendados por farmacêuticos são os medicamentos para tosse $\mathrm{e}$ resfriado, analgésicos, antiinflamatórios, agentes gastrointestinal, antiácidos, suplementos nutricionais e vitaminas, antifúngicos, medicamentos dermatológicos,

produtos oftalmológicos, higiene oral, laxantes, correlatos para primeiro socorros, contraceptivos e produtos de saúde da mulher ${ }^{(19)}$.

Para a consolidação da semiologia farmacêutica se faz necessário que o farmacêutico esteja em constante atualização para adquirir informações sobre estes produtos em fontes confiáveis, a fim de ajudar os pacientes a tomar decisões seguras sobre suas compras de $\operatorname{MIPs}^{(19)}$. Sabe-se que no Reino Unido alguns farmacêuticos já estão oferecendo serviços adicionais, como conselho pró ativo de promoção da saúde, serviços de informações nas farmácias ou consultas com pacientes individuais ${ }^{(20)}$. Os MIP's devem ser seguros e efetivos, trazendo benefícios a sociedade quando usado de forma adequada pela automedicação responsável, uma vez que em busca de qualidade de vida da população consegue tratar suas pequenas enfermidades com praticidade e ainda acarreta diminuição de custos para o individuo e para o sistema de saúde ${ }^{(21)}$.

Em 2009, um levantamento realizado pelo "Guía Terapéutica en Atención Primaria Basada en la Selección Razonada del Medicamento", reassaltou a importância da escolha na seleção dos medicamentos, pois novos fármacos são lançados a cada dia e milhares de 


\section{REF-ISSN1808-0804 Vol.XI (2),55-69, 2014.}

artigos com ensaios clínicos estatisticamente significativos são publicados todos os anos. A elaboração de um método de prescrição fundamentado no uso racional de medicamentos, de forma a ordenar as ideias e direcionar os farmacêuticos à tomada de decisões vem sendo aconselhado pela $\mathrm{OMS}^{(22)}$.

Um estudo realizado no norte da Índia observou que muitos medicamentos que só podem ser comprados com receita médica em países desenvolvidos são considerados medicamentos isentos de prescrição nos países em desenvolvimento ${ }^{(23)}$. Na Finlandia os pacientes tem facil acesso aos medicamentos isentos de prescrição, em farmácias comunitárias, para tratar suas doenças menores. Desde 1983, a Lei de Medicamentos da Finlândia no 395, $\S 5$ 0, 895/96, delega aos farmacêuticos a oferecer "aconselhamento farmacêutico", sendo sua responsabilidade na prestação de terapia medicamentosa, para alcançar resultados concretos que melhorem a qualidade de vida do paciente, assegurando que o mesmo compreendeu as instruções e advertências de sua medicação( ${ }^{(2)}$.

No Canadá a campanha "drugbenefit program" permite que os membros das Forças Armadas canadenses recebam medicamentos de venda livre nas farmácias sem consultar um médico e o farmacêutico é reembolsado pelo custo da medicação como uma taxa de serviço. O programa tem sido benéfico para reduzir a carga de trabalho dos médicos em tratamento de doenças menores e minimizar os custos associados com visitas desnecessárias ao médico. Em Nottingham, para melhorar o acesso aos medicamentos foi implantado um esquema para atender doenças menores "Pharmacy First Minor Ailments Scheme (PFS)", em dezembro de 2003, onde as pessoas inscritas no programa "Primary Care Trust" (PCT) receberam gratuitamente as medicações para atenção primária(24).

$\mathrm{Na}$ Austrália a prescrição farmacêutica se restringe aos medicamentos isentos de prescrição. Estudos realizados em 2011 para explorar as questões da ampliação da prescrição farmacêutica, concluiu que do ponto de vista dos farmacêuticos e pacientes a prescrição farmacêutica deveria ser ampliada para beneficiar a população(25). Os farmacêuticos interagem com $80 \%$ dos usuários de sistema de saúde paliativos, sendo eles pacientes ou acompanhantes, o constante aprendizado do farmacêutico é essencial para garantir um atendimento correto e adequado ao paciente. Para isso, sistemas computadorizados, são elaborados para fornecer informações sobre determinadas 
PEDROSO, T. M.; MASTROIANNI, P. C.; SANTOS, J. L.

REF-ISSN1808-0804 Vol.XI (2),55-69, 2014.

patologias auxiliando assim 0

profissional farmacêutico $(25)$. A

interveção farmacêutica deve ser realizada em colaboração com o médico, pois é dele o papel fundamental no diagnóstico ${ }^{(25)}$.

Dois estudos realizados na Austrália e na Suíça, indicou que os farmacêuticos recebem treinamentos para aconselhar e orientar os usuários de medicamentos quanto ao uso seguro e correto, promovendo a automedicação responsável $^{(26-28)}$. Mas na realidade os pacientes se automedicam para diversos sintomas e muitas vezes as indicações para a escolha do medicamento advêm de familiares, amigos e tratamentos prévios, sendo que, a participação do farmacêutico no processo da automedicação é verificada em apenas alguns $\operatorname{casos}^{(17,30-31)}$.

Em 2012 um estudo apresentado por Fletcher $^{(32)}$ e colaboradores demonstrou que a intervenção do farmacêutico e profissionais de enfermagem na avaliação e otimização do uso de fármacos, alcançou melhorias terapêuticas, e o aconselhamento fornecido por profissionais da saúde em relação ao uso correto e seguro de fármacos contribui significativamente para a redução das taxas de morbidade e mortalidade.

No Brasil a RDC n.0 357/2001 aprova o regulamento técnico das
"Boas Práticas de Farmácia", sequencialmente na Resolução Brasileira - RDC no 138/2003, os farmacêuticos encontram uma lista de Grupos e Indicação Terapêuticas Especificadas - GITE, esses fármacos podem ser vendidos sem prescrição médica. Logo, todos os medicamentos cujos grupos terapêuticos e indicações terapêuticas não estão descritos no GITE são de venda sob prescrição médica. A resolução no 499/2008, do Conselho Federal de Farmácia que dispõe sobre a prestação de serviços farmacêuticos, em farmácias e drogarias, e dá outras providências, bem como a Resolução no 44, de 17 de agosto de 2009 que dispõe sobre Boas Práticas Farmacêuticas para o controle sanitário do funcionamento, da dispensação e da comercialização de produtos e da prestação de serviços farmacêuticos em farmácias e drogarias e dá outras providências, são documentos que auxiliam 0 farmacêutico para melhor orientar o paciente frente à tomada de decisões ao escolher um tratamento, procurando padronizar os tipos de medicamentos usados como primeira escolha para as doenças consideradas de transtornos menores, especialmente na atenção primária à saúde ${ }^{(32-36)}$.

Ações educativas para todos os farmacêuticos são desenvolvidos pelo Conselho Federal de Farmácia sendo a maioria oferecida gratuitamente como 
PEDROSO, T. M.; MASTROIANNI, P. C.; SANTOS, J. L.

REF-ISSN1808-0804 Vol.XI (2),55-69, 2014.

os cursos de educação continuada, disponibilizados em Fascículos

"Farmácia Estabelecimento de Saúde", que tratam de temas técnicos de interesse da profissão farmacêutica. Cursos presenciais são periodicamente realizados nas sedes dos conselhos regionais de farmácia em diferentes regiões do país, o farmacêutico também pode optar por assistir via internet cursos, palestras e seminários realizados fora de sua localidade. No Brasil, recentemente aprovada, a resolução do Conselho Federal de Farmácia - CFF no 585 de 29 de agosto de 2013 e no 586 de 29 de Agosto de 2013 vem regulamentar as atribuições clínicas do farmacêutico, bem como, implantar e regulamentar a prescrição farmacêutica. Este documento autoriza os farmacêuticos a prescreverem medicamentos e outros produtos com finalidade terapêutica, cuja dispensação não exija prescrição médica, para o tratamento dos transtornos menores ${ }^{(37-38)}$. No inicio de 2014 campanhas comemorativas ao dia nacional do farmacêutico (20 de Janeiro) foram veiculadas em rede nacional tendo como tema: "Onde tem medicamento tem que ter farmacêutico" com 0 intuito de conscientizar a população da importância deste profissional para a sociedade. Ressalta-se considerar que a prestação de serviços farmacêuticos com qualidade possivelmente fidelizará os clientes e consequentemente haverá um maior retorno financeiro aos proprietários.

\section{DISCUSSÃO}

A análise deste trabalho corrobora a idéia de que existe um movimento de mobilização mundial, no sentido de capacitação do farmacêutico para o tratamento de doenças menores, considerando esse fato como um avanço necessário para beneficiar a população e diminuir custos atrelados a forma convencional de atendimento, que se mostra menos eficiente do ponto de vista econômico. Em alguns países, os farmacêuticos já têm autonomia prescritiva para auxiliar os pacientes que buscam nas farmácias os medicamentos isentos de prescrição médica, desencadeando o tratamento imediato de seus sinais e sintomas prevenindo assim uma possível piora do seu quadro clínico. 0 farmacêutico desempenha a função de acompanhamento, orientando e verificando adesão do tratamento dos usuários. Nos casos de grupos de risco e doenças de maior complexidade, os pacientes são encaminhados às especialidades médicas. Em 2011, o Canadá aderiu a este modelo, de forma que os farmacêuticos daquele país passaram a gozar de maior grau de autonomia prescritiva. Mas um estudo conduzido por Awad e colaboradores ${ }^{(39)}$ verificou que aproximadamente $75 \%$ dos 
REF-ISSN1808-0804 Vol.XI (2),55-69, 2014.

farmacêuticos relataram que há necessidade de maior aprendizado em como promover a saúde em farmácias e drogarias e a falta de tempo é o principal fator que dificulta a promoção da saúde no nível primário de atenção.

No Brasil, o farmacêutico ainda não tem atuação destacada no acompanhamento da utilização de medicamentos, na prevenção e promoção da saúde, e é pouco reconhecido como profissional de saúde tanto pela sociedade quanto pela equipe de saúde. Observa-se uma postura passiva dos farmacêuticos perante $o$ principal serviço que deveria ser oferecido nas farmácias, à orientação quanto ao uso correto e seguro, contribuindo para o uso racional de medicamentos, prevenindo agravos à saúde do paciente e a sobrecarga das instituições de saúde. Ademais, fomenta o questionamento do ato de dispensação ter sido delegado aos balconistas, cujo conhecimento técnico é restrito, pelo fato do nível de escolaridade destes atendentes abrangerem muitas vezes apenas o ensino médio. Portanto, a ação do farmacêutico como promotor da saúde e do auto-cuidado e, principalmente, de educador em relação ao uso seguro do medicamento, deixa a desejar devido à demanda de trabalhos burocráticos nas drogarias, de modo geral, o principal serviço prestado nas farmácias e drogarias é a dispensação de medicamentos, sendo a qualidade dessa prática considerada abaixo do padrão, uma vez que os afazeres técnicos toma grande parte da sua carga horária de trabalho e acabam não fazendo a sua real função que é exercer os seus conhecimentos sobre medicamentos e assim auxiliar na promoção da saúde $^{(40}$. Esta omissão pode resultar em agravos à saúde dos usuários de medicamentos.

Embora estejamos muito aquém dos níveis atingidos por países europeus, Estados Unidos e Canadá, é possível verificar que existe um grande interesse brasileiro, tanto da comunidade acadêmica, como do Conselho Federal de Farmácia - CFF desse País, no sentido de viabilizar as possibilidades para a implementação dessa prática. Ressaltam-se neste debate as dificuldades encontradas, relativas à qualificação dos profissionais, devido à falta de conteúdos curriculares relacionados à semiologia farmacêutica e farmacoterapia nos cursos de graduação e pós-graduação. Essas estratégias de aprendizagem ampliam a capacitação para atuação dos profissionais farmacêuticos. No Brasil, a inclusão de disciplinas de Semiologia Farmacêutica, Atenção Farmacêutica e Farmacovigilância é recente em algumas universidades do país, para promover a capacitação e 
PEDROSO, T. M.; MASTROIANNI, P. C.; SANTOS, J. L.

REF-ISSN1808-0804 Vol.XI (2),55-69, 2014.

habilitação desses profissionais, mas há poucos profissionais aptos a desenvolverem a atividades clínicas, e específicas para o tratamento de determinados sinais e sintomas.

Com esta formação, o farmacêutico poderá colocar em prática a semiologia farmacêutica, como um segmento dentro da atenção farmacêutica, que busca através dos sinais e sintomas dos pacientes investigar a efetividade e segurança de medicamentos em uso ou não, e auxiliá-los na resolução de problemas de saúde relacionados à farmacoterapia. É necessário um monitoramento desses pacientes, com base na fidedignidade dos mesmos em relação à terapia proposta pelo farmacêutico e a sua percepção com relação aos sinais e sintomas, pois somente desta forma é possível avaliar se a terapia proposta pelo profissional está sendo bem sucedida.

\section{CONCLUSÃO}

A semiologia farmacêutica é uma nova prática profissional que visa a identificação de sinais e sintomas em distúrbios menores para posterior decisão terapêutica do medicamento isento de prescrição (MIP) mais efetivo e seguro. A seleção adequada do MIP, sob responsabilidade farmacêutica, tem como finalidade a promoção da saúde e prevenção de problemas relacionados com uso de medicamentos. A semiologia farmacêutica vem sendo encorajada em diferentes países, entretanto, desafios à sua implementação ainda devem ser superados, como por exemplo, formação profissional. Critérios de seleção de medicamentos devem ser conhecidos e pautados em protocolos clínicos para garantir uso adequado do medicamento. Sendo a farmácia comunitária uma das portas de acesso da população à saúde, o desenvolvimento dessa prática contribui para melhor do sistema de saúde do país, ampliando acesso da população e permitindo resolutibilidade de agravos menores na farmácia da comunidade.

\section{AGRADECIMENTOS}

Apoio financeiro: CAPES, CNPQ, FAPESP, PROEXT-2010 - MEC.

\section{REFERÊNCIAS}

1. WHO - WORLD HEALTH ORGANIZATION Promoting Rational use of medicines: core components. Policy Perspective on Medicines, Geneva, 2002.

2. Kansanaho $\mathrm{H}$, Isonen-Sjo NL, Pietila $\mathrm{K}$, Airaksinen $\mathrm{M}$, Isonen T. Patient counselling profile in a Finnish pharmacy. Patient Educ Couns. 2002;47(1):77-82. 
PEDROSO, T. M.; MASTROIANNI, P. C.; SANTOS, J. L.

REF-ISSN1808-0804 Vol.XI (2),55-69, 2014.

3. Craig DS. Oxymorphone Extended-Release Tablets (Opana ER) For the Management of Chronic Pain. P \& T. 2010;35(6):324-329.

4. FIP - International Pharmaceutical Federation. Guidelines for drug procurement, 1992.

5. Johnson JA, Bootman JL. Drug-related morbidity and mortality: A cost-ofillness model. Arch Intern Med. 1995;155(18):1949-1956.

6. Júnior $D P L$, Kheir N, Abriata JP, Rocha $C E$, Santos $C B$, Pelá IR. Impact of Pharmaceutical Care interventions in the identification and resolution of drugrelated problems and on quality of life in a group of elderly outpatients in Ribeirão Preto (SP), Brazil. Ther Clin Risk Manag. 2007;3(6):989-998.

7. Macbryde CM, Bralcklow R. Sinais e sintomas: fisiopatologia aplicada e interpretação clínica. 5. ed. Rio de Janeiro: Guanabara Koogan, 1975. p.1-16.

8. Manuila L, Manuila A, Nicoulin M. Dicionário médico Andrei. 7.ed. São Paulo: Organização Andrei, 1997.

9. Byrd GD. Can the profession of pharmacy serve as a model for health informationist professionals?. J Med Libr Assoc. 2002;90(1): 68-75.

10. Porteous $T$, Ryan $M$, Bond $C M$, Hannaford $P$. Preferences for self-care or professional advice for minor illness: a discrete choice experiment. $\mathrm{Br}$ J Gen Pract. 2006;56(533):911-917.

11. Lezley $\mathbf{A H}$, Carmel MH. Public's views on making decisions about over-thecounter medication and their attitudes towards evidence of effectiveness: A crosssectional questionnaire study. Pharm World Sci. 2011;83(3):345-351.

12. Arrais PSD, Coelho HLL, Batista MMCDS, Carvaljp ML, Righi RE, Arnai JM. Aspects of self-medication in Brazil. Rev Saude Publica. 1997;31(1):71-77. 
PEDROSO, T. M.; MASTROIANNI, P. C.; SANTOS, J. L.

REF-ISSN1808-0804 Vol.XI (2),55-69, 2014.

13. WHO - WORLD HEALTH ORGANIZATION. Guidelines for the medical assessment of drugs for use in self-medication, Copenhagen, 1986.

14. Dalgalarrondo P. Psicopatologia e Semiologia dos Transtornos Mentais. Porto Alegre: Editora Artmed; 2000.

15. Mendes EV. O cuidado das condições crônicas na atenção primária à saúde: o imperativo da consolidação da estratégia da saúde da família. Brasília: Organização Pan-Americana da Saúde; 2012.

16. Daisy V, John L, David H. How well informed are pharmacy customers in Estonia about minor illnesses and over-the-counter medicines. Medicina (Kaunas). 2007;43(1):70-78.

17. Paudyal V, Hansford D, Cunningham S, Stewart D. Pharmacy assisted patient self care of minor ailments: A chronological review of UK health policy documents and key events 1997-2010. Health Policy. 2011;101(3):253-259.

18. Parmentier H, Golding MDS, Ashworth M, Rowlands G. Community pharmacy treatment of minor ailments in refugees. J Clin Pharm Ther. 2004;29(5):465-469.

19. Pharmacists Take Center Stage in OTC Counseling. US Pharm. 2007;32(7):4-6.

20. Porteous $T$, Ryan M, Bond CM, Hannaford P. Novel provision of pharmacy services to a deprived area: a pharmaceutical needs assessment. Int J Pharm Pract. 2003;11(1):47-54.

21. Marques, LAM. Atenção Farmacêutica Em Distúrbios Menores. 2. Ed. São Paulo:MEDFARMA, 2008.

22. GUÍA TERAPÉUTICA EN ATENCIÓN PRIMARIA BASADA EN LA SELECCIÓN RAZONADA DEL MEDICAMENTO. Necesidad de seleccionar medicamentos: La responsabilidad de elegir. The need to select drugs: the responsibility of choosing. Aten Primaria. 2009;41(12):657-658.

23. Rohit KV, Lalit M, Manisha P. Evaluation of self medication among Professional students in North India: proper statutory drug control must be implemented. Asian J Pharm Clin Res. 2010;3(1):60-64. 
PEDROSO, T. M.; MASTROIANNI, P. C.; SANTOS, J. L.

REF-ISSN1808-0804 Vol.XI (2),55-69, 2014.

24. Pumtong S, Boardman HF, Anderson CW. Pharmacists' perspectives on the Pharmacy First Minor Ailments Scheme. Int J Pharm Pract.2008;16(2):73-80.

25. Kreshnik $H$, Jeffery $H$, Bruce $S$. An expanded prescribing role for pharmacists - an Australian perspective. Australas Med J. 2011;4(4):236-242.

26. Hussainy SY, Marriott JL, Beattie J, Nation RL, Dooley MJ. A palliative cancer care flexible education program for Australian community pharmacists. Am J Pharm Educ. 2010;74(2):24.

27. Krishnan HS, Schaefer M. Evaluation of the impact of pharmacist's advice giving on the outcomes of self-medication in patients suffering from dyspepsia. Pharm World Sci. 2000;22(3):102-108.

28. Hersberger KE, Renggli VP, Nirkko AC, Mathis J, Schwegler K, Bloch KE. Screening for sleep disorders in community pharmacies -evaluation of a campaign in Switzerland. J Clin Pharm Ther. 2006;31(1):35-41.

29. Marklund B, Westerlund T, Brastad JO, SJöblom M. Referrals of dyspeptic self-care patients from pharmacies to physicians, supported by clinical guidelines. Pharm World Sci. 2003;25(4):168-172.

30. Albarrán KF, Zapata LV. Analysis and quantification of self-medication patterns of customers in community pharmacies in southern Chile. Pharm World Sci. $2008 ; 30(6): 863-868$.

31. Williams A, Scadding G. Is reliance on self-medication and pharmacy care adequate for rhinitis patients?. Int J Clin Pract.2009;63(1):98-104.

32. Fletcher J, Hogg W, Farrell B, Woodend K, Dahrouge S, Lemelin J, Dalziel W. Effect of nurse practitioner and pharmacist counseling on inappropriate medication use in family practice. Can Fam Physician. 2012;58(8):862-868.

33. BRASIL. Conselho Federal de Farmácia. Resolução no 357 de 20 de Abril de 2001. Aprova o regulamento técnico das Boas Práticas de Farmácia. Diário Oficial da União, Brasília, 20 de abril 2001. 
PEDROSO, T. M.; MASTROIANNI, P. C.; SANTOS, J. L.

REF-ISSN1808-0804 Vol.XI (2),55-69, 2014.

34. BRASIL, Ministério da Saúde. Resolução da Diretoria Colegiada - RDC no 138 de 29 de Maio de 2003. Dispõe sobre o enquadramento na categoria de venda de medicamentos. Diário Oficial da União, Brasília, 02 de junho 2003.

35. BRASIL. Conselho Federal de Farmácia. Resolução no 499 de 17 de dezembro de 2008. Dispõe sobre a prestação de serviços farmacêuticos, em farmácias e drogarias, e dá outras providências. Diário Oficial da União, Brasília, 23 de dezembro 2008.

36. BRASIL. Agência Nacional de Vigilância Sanitária Resolução no 44, de 17 de agosto de 2009. Dispõe sobre Boas Práticas Farmacêuticas para o controle sanitário do funcionamento, da dispensação e da comercialização de produtos e da prestação de serviços farmacêuticos em farmácias e drogarias e dá outras providências. Diário Oficial da União, Brasília, 17 de agosto 2009.

37. BRASIL. Conselho Federal de Farmácia. Resolução n० 585 de 29 de agosto de 2013 Ementa: Regulamenta as atribuições clínicas do farmacêutico e dá outras providências. Diário Oficial da União, Brasília, 25 de setembro 2013.

38. BRASIL. Conselho Federal de Farmácia. Resolução no 586 de 29 de Agosto de 2013. Regula a prescrição farmacêutica e dá outras providências. Diário Oficial da União, Brasília, 25 de setembro 2013.

39. Awad A, Abahussain E. Health promotion and education activities of community pharmacists in Kuwait. Pharm World Sci. 2010;32(2):146-153.

40. Castro MS, Correr CJ. Pharmaceutical care in community pharmacies: practice and research in Brazil. Pharmacotherapy. 2007;41(9):1486-1493. 